\title{
Perfil da população cadastrada em uma Unidade Básica de Saúde da Família: a importância da classificação de risco familiar
}

\author{
Sandy Borges Rosa, Fernanda de Jesus Santiago, Luciana Saraiva da Silva
}

\section{RESUMO}

A Atenção Primária à Saúde (APS) é considerada o primeiro nível de atenção em saúde e tem na Saúde da Família sua estratégia prioritária para expansão e consolidação. $O$ objetivo do estudo foi descrever o perfil e a classificação de risco familiar dos usuários de uma Unidade Básica de Saúde da Família em Uberlândia, Minas Gerais. Trata-se de um estudo transversal, com dados coletados dos Sistemas de Informações, nos anos 2017 e 2018. Foram analisadas variáveis sociodemográficas, classificação dos grupos populacionais e risco familiar. Para a classificação de risco familiar foram considerados fatores socioeconômicos (grau de alfabetização do chefe da família, renda e abastecimento de água) e fatores clínicos (grau de risco dos seguintes grupos/condições: gestante, criança, hipertensão, diabetes, doença renal crônica ou outras condições crônicas de alto risco). Foram avaliados 1553 usuários, sendo 70,6\% do sexo feminino. A faixa etária predominante foi entre 40 e 59 anos $(33,23 \%)$ e a menos atendida foi a de adolescentes entre 14 e 17 anos (1,55\%). Em relação à classificação de risco, observou-se maiores prevalências de hipertensão com médio risco, diabetes de alto risco e tuberculose de baixo risco. $O$ estudo evidencia a importância do fortalecimento de políticas públicas, que visem à promoção e monitoramento das condições de saúde na $A B$.

Palavras-chave: atenção primária à saúde, fator de risco, doença crônica.

\section{ABSTRACT}

Primary Health Care (PHC) is considered the first level of health care and has in Family Health its priority strategy for expansion and consolidation. The objective of the study was to describe the profile and family risk classification of users of the Family Health Unit in Uberlândia, Minas Gerais. This is a cross-sectional study, with data collected from the municipality's Information Systems, during the years 2017 and 2018. Sociodemographic variables, classification of population groups and family risk were analyzed. For the classification of family risk, socioeconomic factors (degree of literacy of the head of the household, income and water supply) and clinical factors (degree of risk of the following groups/conditions: pregnant woman, child, hypertension, diabetes, chronic kidney disease or other high-risk conditions). A total of 1553 users were evaluated, $\mathbf{7 0 . 6 \%}$ of whom were female. The predominant age group was that of adults between 40 and 59 years old (33.23\%) and the least attended was that of adolescents between 14 and 17 years old (1.55\%). Regarding the risk classification, there was a higher prevalence of medium-risk hypertension, high-risk diabetes and low-risk tuberculosis. The study highlights the importance of strengthening public policies, aimed at promoting and monitoring health conditions in PHC.

Keywords: primary health care, risk factors, chronic disease.
Revista da Rede APS 2021

Publicada em: 20/09/2021

DOI:10.14295/aps.v3i2.175

Sandy Borges Rosa

(Universidade Federal de

Uberlândia, Uberlândia, MG, Brasil)

Fernanda de Jesus Santiago (Universidade Federal de Uberlândia, Uberlândia, MG, Brasil)

Luciana Saraiva da Silva (Universidade Federal de Uberlândia, Uberlândia, MG, Brasil)

\section{Correspondência para:}

Luciana Saraiva da Silva lucianassnut@gmail.com 


\section{INTRODUÇÃO}

A atenção primária à saúde (APS) é considerada o primeiro nível de atenção em saúde e tem na Saúde da Família sua estratégia prioritária para expansão e consolidação. A APS tem por finalidade oferecer cuidado integral a saúde da população, por meio da vigilância dos determinantes de risco, monitoramento e acompanhamento das individualidades e promoção do autocuidado (BRASIL, 2012). Neste contexto, uma das principais atividades desenvolvidas pelas equipes de Saúde da Família é a estratificação ou classificação de risco, por meio da investigação e detecção de fatores individuais ou coletivos que interferem no estado de saúde da população (BRASIL, 2012).

A identificação dos riscos de cada usuário e de sua família ajuda as equipes a adequar as estratégias, tanto individuais como coletivas, conforme as necessidades da população, além de utilizar melhor os recursos do serviço (MENDES, 2012; BRASIL, 2012). O processo demanda uma profunda investigação dos hábitos familiares, comportamentos, idade, sexo, estado de saúde inicial que pode vir a ocasionar um agravo da condição de saúde, além das relações psicossociais que podem interferir no estado emocional e físico do indivíduo (BRASIL, 2012; BRASIL, 2014).

Desta forma, a partir das características identificadas, os indivíduos e suas famílias são classificados em baixo (leve), médio (moderado), alto risco ou muito alto risco (PLANO DIRETOR APS, 2010; MENDES, 2012; SECRETARIA DE SAÚDE, 2018). Neste caso, estratificar significa reconhecer que as pessoas têm diferentes graus de risco/vulnerabilidades e, portanto, têm diferentes necessidades (MENDES, 2012). A gravidade da condição é resultado da complexidade do problema e sua interferência na qualidade de vida da pessoa e do risco de ocorrer um evento que cause morbidade ou mortalidade (BRASIL, 2014).

Nesta perspectiva, considera-se então como alto risco quanto menor for as condições financeiras, o grau de escolaridade, as condições de moradia, o acesso ao saneamento básico, quando a família apresentar membros com doenças crônicas ou que necessitem de um acompanhamento e cuidado especial. Ou seja, o estrato de alto risco, representa pessoas que apresentam insuficiente capacidade para o autocuidado e possuem enfermidade crônica muito complexa, demandando atenção mais concentrada e individual por parte dos profissionais. Já o estrato de baixo risco, que representa a situação de menor risco/vulnerabilidade, refere-se às pessoas que possuem apenas os fatores de risco ligados a comportamentos e a estilo de vida, não apresentando doença estabelecida, o que é equivalente à maioria da população (PLANO DIRETOR APS, 2010; MENDES, 2012; SECRETARIA DE SAÚDE, 2018).

Destaca-se ainda a importância da classificação de risco, especialmente quando se considera as crescentes prevalências e incidências das doenças crônicas não transmissíveis (DCNT), que são um dos maiores problemas de saúde pública do Brasil e do mundo (OMS, 2018; VIGITEL, 2019). Assim sendo, tomar conhecimento do perfil da população cadastrada na APS é o primeiro passo para planejar ações e programas que reduzam à ocorrência de doenças, garantindo adequado atendimento às demandas e necessidades do público e dessa forma melhorar a saúde da população. Portanto, o objetivo do presente estudo foi descrever o perfil e a classificação de risco familiar dos usuários cadastrados em uma Unidade Básica de Saúde da Família (UBSF) em Uberlândia, Minas Gerais.

\section{MATERIAIS E MÉTODOS}

\section{Local de estudo}

O estudo foi realizado em Uberlândia, que se localiza na mesorregião do Triângulo Mineiro. Segundo dados do IBGE (2018), a população estimada de Uberlândia em 2018 era de 683.247 habitantes, sendo 666.480 residentes da zona urbana e 16.767 da zona rural.

De acordo com a Prefeitura Municipal de Uberlândia, em 2017, o município possuía 74 
equipes de Saúde da Família, com 68 unidades na zona urbana e seis na zona rural. Em novembro de 2017, a população coberta pela Saúde da Família foi estimada em 255.300 habitantes, correspondendo a 38,3\% da população total do município (DATASUS, 2017).

O estudo foi realizado em uma UBSF, localizada no setor Leste de Uberlândia, que contava com seis equipes de saúde da família.

\section{Instrumentos e coleta de dados}

Os dados dos pacientes cadastrados na UBSF foram coletados durante os anos de 2017 e 2018, por meio dos Sistemas de Informações do município referentes a Ficha de Cadastro Individual e/ou Ficha de Cadastramento Familiar e pelos prontuários eletrônicos (Sistema Fast Medic).

Foram analisadas variáveis sociodemográficas (sexo, idade), classificação dos grupos populacionais e epidemiológicos (criança/adolescente, diabético tipo I, diabético tipo II, hanseníase, hipertenso, hipertenso e diabético tipo I, hipertenso e diabético tipo II, saúde da mulher e tuberculose) e classificação de risco (baixo, médio, alto ou muito alto risco). Foram incluídos todos os participantes com dados disponíveis nos Sistemas de Informações.

Com o intuito de classificar as famílias, conforme recomendação do Plano Diretor da Atenção Primária à Saúde (PLANO DIRETOR APS, 2010), inicialmente os profissionais identificaram e avaliaram os condicionantes primários do âmbito social (fatores socioeconômicos), sendo eles, o grau de alfabetização do chefe da família, a renda (considerando situação de extrema pobreza a inclusão no Programa Bolsa Família) e abastecimento de água. Foi considerada de risco a família que apresentou um ou mais dos fatores de risco listados, sendo um ponto para cada um dos três fatores de risco (SECRETARIA DE SAÚDE, 2018).

Outro aspecto avaliado para a classificação de risco foram as doenças crônicas, sendo considerada de risco a família, em que um ou mais de seus integrantes apresentaram uma ou mais das seguintes condições ou patologias por ciclo de vida: gestante de alto risco, criança de alto risco, hipertenso de alto ou muito alto risco, diabético de alto ou muito alto risco, indivíduo com Doença Renal Crônica de alto ou muito alto risco ou outras condições crônicas de alto risco definidas como prioritárias pela equipe de saúde. A pontuação referente a essa classificação foi de um quando apenas um indivíduo possuía uma dessas doenças, dois para aquela família que apresentava dois ou mais indivíduos com alguma dessas condições clínicas, ou ainda foi pontuado três quando se tinha um ou mais indivíduos com duas ou mais dessas doenças (SECRETARIA DE SAÚDE, 2018).

Em seguida foi realizada a soma final dos pontos adquiridos, considerando cada um dos critérios analisados no questionário (socioeconômicos e clínicos). Por fim, a família foi classificada como "sem risco" quando a pontuação total foi igual a zero pontos; em baixo risco quando a pontuação total foi de um ponto; em médio risco quando a pontuação total foi de dois ou três pontos e em alto risco quando a pontuação total foi maior ou igual a quatro pontos (SECRETARIA DE SAÚDE, 2018).

\section{Análise dos dados}

Para análise descritiva das variáveis foram apresentadas tabelas de frequência absoluta e relativa. Utilizou-se o software SPSS for Windows (Version 20.0; SPSS Inc, Chicago).

\section{Aspectos Éticos}

O projeto foi aprovado pela comissão de ética em pesquisa com seres humanos da Universidade Federal de Uberlândia, sob parecer número: 2.818.549.

Foi aprovada a dispensa do uso de Termo de Consentimento Livre e Esclarecido (TCLE) por ser um estudo observacional, que empregou apenas informações de prontuários médicos e/ou sistemas de informação institucionais sem utilização de material biológico. De acordo com a resolução №. 196/96 do Conselho Nacional de Saúde, que regulamenta as pesquisas 
envolvendo seres humanos, foi garantida a confidencialidade das informações e o anonimato da identificação dos participantes do estudo.

\section{RESULTADOS}

Foram avaliados 1553 usuários da UBSF. Conforme evidenciado na Tabela 1, a faixa etária mais atendida na unidade foi de adultos entre 40 e 59 anos $(33,23 \%)$, seguida de 60 anos ou mais (18,8\%). A faixa etária menos atendida na unidade foi a de adolescentes entre 14 e 17 anos $(1,55 \%)$.

Em todas as faixas etárias analisadas, houve maior prevalência do sexo feminino, sendo que mais de $70 \%$ da amostra foi composta por mulheres.

Tabela 1 - Faixa etária dos indivíduos cadastrados em uma UBSF, Uberlândia-MG, 2017-2018

\begin{tabular}{cccc}
\hline Faixa etária & Sexo feminino & Sexo masculino & Total \\
& $\mathbf{n}$ & $\mathbf{n}$ & $\mathbf{n}(\%)$ \\
\hline 0 a 5 anos & 104 & 95 & $199(12,81)$ \\
14 a 13 anos 17 anos & 59 & 57 & $116(7,47)$ \\
18 a 24 anos & 15 & 9 & $24(1,55)$ \\
25 a 39 anos & 120 & 10 & $130(8,37)$ \\
40 a 59 anos & 253 & 23 & $276(17,77)$ \\
60 anos ou mais & 368 & 148 & $516(33,23)$ \\
\hline Total & 178 & 114 & $292(18,80)$ \\
\hline
\end{tabular}

Fonte: elaborada pelos autores

Do total de pessoas avaliadas, 41,53\% ( $n=641)$ pertenciam ao grupo de saúde da mulher, 29,36\% ( $n=454)$ ao grupo de hipertensos, $22,41 \%$ ( $n=349)$ ao grupo criança/adolescente e $15 \%(n=232)$ ao grupo de tuberculose, que foram os grupos mais prevalentes.

Foi possível observar grande prevalência das DCNT, destacando hipertensão arterial (HA) e diabetes mellitus (DM) tipo II. No grupo de HA, a prevalência foi maior no sexo feminino $(17,71 \%)$ e na classificação de médio risco (16,29\%). Já em relação ao DM tipo II, encontrou-se uma prevalência de $10,5 \%$ somando os diabéticos tipo II e os hipertensos e diabéticos tipo II, sendo a maioria de alto risco $(6,96 \%)$.
No grupo de saúde integral da mulher, notou-se a maioria como não gestante, em idade fértil e sem risco $(23,44 \%)$, seguida por mulheres em climatério/menopausa (11,14\%).

Outro grupo que chamou atenção em relação à prevalência foi o de tuberculose, já que cerca de $15 \%(n=232)$ da população cadastrada na unidade apresentavam esta doença. A maior prevalência foi encontrada no sexo feminino $(9,9 \%)$, sendo que $14,75 \%$ do total apresentava baixo risco.

Os demais grupos podem ser observados na Tabela 2 
Tabela 2 - Análise descritiva dos grupos cadastrados em uma UBSF, segundo classificação de risco e sexo. Uberlândia-MG, 2017-2018

\begin{tabular}{|c|c|c|c|c|}
\hline \multirow{2}{*}{$\begin{array}{c}\text { Grupos e classificações de } \\
\text { risco }\end{array}$} & \multirow{2}{*}{$\begin{array}{c}n \\
(n=1553)\end{array}$} & \multirow[t]{2}{*}{$\%$} & \multicolumn{2}{|c|}{ Sexo } \\
\hline & & & $\begin{array}{c}\text { Feminino } \\
\mathrm{n}(\%)\end{array}$ & $\begin{array}{c}\text { Masculin } \\
\text { o } \\
\text { n (\%) }\end{array}$ \\
\hline Criança/Adolescente & 349 & $\begin{array}{c}22,41 \\
(I C 95 \% 20,4-24,5)\end{array}$ & $\begin{array}{c}197 \\
(12,69)\end{array}$ & $\begin{array}{c}152 \\
(9,79)\end{array}$ \\
\hline Sem risco & 69 & 4,44 & $32(2,06)$ & $37(2,38)$ \\
\hline Risco não apresentado & 31 & 2 & $27(1,74)$ & $4(0,26)$ \\
\hline Baixo risco & 204 & 13,14 & $111(7,15)$ & $93(5,99)$ \\
\hline Médio risco & 11 & 0,71 & $7(0,45)$ & $4(0,26)$ \\
\hline Alto risco & 34 & 2,19 & $20(1,29)$ & $14(0,90)$ \\
\hline Diabético tipo I & 3 & $\begin{array}{c}0,19 \\
(I C \text { 95\% 0,06 - 0,05) }\end{array}$ & $1(0,06)$ & $2(0,12)$ \\
\hline Baixo risco & 2 & 0,13 & $1(0,06)$ & $1(0,06)$ \\
\hline Muito alto risco & 1 & 0,06 & 0 & $1(0,06)$ \\
\hline Diabético tipo II & 40 & $\begin{array}{c}2,58 \\
(I C 95 \% 1,89-3,49) \\
\end{array}$ & $18(1,16)$ & $22(1,42)$ \\
\hline Baixo risco & 17 & 1,09 & $9(0,58)$ & $8(0,52)$ \\
\hline Médio risco & 7 & 0,45 & $2(0,13)$ & $5(0,32)$ \\
\hline Alto risco & 11 & 0,71 & $4(0,26)$ & $7(0,45)$ \\
\hline Muito alto risco & 5 & 0,32 & $3(0,19)$ & $2(0,13)$ \\
\hline Hanseníase & 5 & $\begin{array}{c}0,32 \\
(I C 95 \% 0,13-0,77) \\
\end{array}$ & $4(0,26)$ & $1(0,06)$ \\
\hline $\begin{array}{l}\text { Multibacilar (risco não } \\
\text { apresentado) } \\
\text { Paubacilar (risco não } \\
\text { apresentado) }\end{array}$ & $\begin{array}{l}1 \\
4\end{array}$ & $\begin{array}{l}0,06 \\
0,26\end{array}$ & $\begin{array}{c}0 \\
4(0,26)\end{array}$ & $\begin{array}{c}1(0,06) \\
0\end{array}$ \\
\hline Hipertenso & 454 & $\begin{array}{c}29,36 \\
(I C 95 \% 27,1-31,6)\end{array}$ & $\begin{array}{c}274 \\
(17,71) \\
\end{array}$ & $\begin{array}{c}180 \\
(11,59)\end{array}$ \\
\hline Baixo risco & 91 & 5,86 & $91(5,86)$ & 0 \\
\hline Médio risco & 252 & 16,29 & $148(9,59)$ & 104 \\
\hline Alto risco & & & & $(6,70)$ \\
\hline Muito alto risco & 63 & 4,06 & $20(1,29)$ & $43(2,77)$ \\
\hline & 48 & 3,09 & $15(0,97)$ & $33(2,12)$ \\
\hline Hipertenso e Diabético tipo I & 1 & $\begin{array}{c}0,06 \\
(\text { IC } 95 \% 0,009-0,45)\end{array}$ & 0 & $1(0,06)$ \\
\hline Alto risco & 1 & 0,06 & 0 & $1(0,06)$ \\
\hline Hipertenso e Diabético tipo II & 123 & $\begin{array}{c}7,92 \\
(\text { IC 95\% 6,67 - 9,37) }\end{array}$ & $76(4,89)$ & $47(3,02)$ \\
\hline Alto risco & 97 & 6,25 & $62(3,99)$ & $35(2,25)$ \\
\hline Muito alto risco & 26 & 1,67 & $14(0,90)$ & $12(0,77)$ \\
\hline Saúde integral da mulher & 641 & $\begin{array}{c}41,53 \\
\text { (IC 95\% 39,1-44) }\end{array}$ & $\begin{array}{c}641 \\
(41,28) \\
\end{array}$ & - \\
\hline $\begin{array}{l}\text { Climatério/Menopausa } \\
\text { Gestação de baixo risco }\end{array}$ & 173 & 11,14 & $\begin{array}{c}173 \\
(11,14)\end{array}$ & - \\
\hline Gestação de risco & 13 & 0,84 & $13(0,84)$ & - \\
\hline intermediário & 12 & 0,77 & $12(0.77)$ & - \\
\hline Gestação de alto risco & 2 & 0,13 & $2(0,13)$ & - \\
\hline $\begin{array}{l}\text { Não gestante, idade fértil, } \\
\text { sem risco }\end{array}$ & 364 & 23,44 & $\begin{array}{c}364 \\
(23,44)\end{array}$ & - \\
\hline & 77 & 4,96 & $77(4,96)$ & - \\
\hline
\end{tabular}


Não gestante, idade fértil, com risco

\begin{tabular}{|c|c|c|c|c|}
\hline Saúde mental & 66 & $\begin{array}{c}4,25 \\
\text { (IC 95\% 3,35 - 5,37) }\end{array}$ & $47(3,03)$ & $19(1,2)$ \\
\hline Sem risco & 41 & 2,64 & $31(2.0)$ & $10(0,64)$ \\
\hline Risco não apresentado & 16 & 1,03 & $11(0,71)$ & $5(0,32)$ \\
\hline $\begin{array}{l}\text { Acompanhamento no CAPS, } \\
\text { baixo risco }\end{array}$ & 2 & 0,13 & $1(0,06)$ & $1(0,06)$ \\
\hline $\begin{array}{l}\text { Acompanhamento no CAPS, } \\
\text { médio risco }\end{array}$ & 1 & 0,06 & 0 & $1(0,06)$ \\
\hline $\begin{array}{l}\text { Acompanhamento no CAPS, } \\
\text { alto risco }\end{array}$ & 5 & 0,32 & $4(0,26)$ & $1(0,06)$ \\
\hline $\begin{array}{l}\text { Acompanhamento no CAPS, } \\
\text { muito alto risco }\end{array}$ & 1 & 0,06 & 0 & $1(0,06)$ \\
\hline Tuberculose & 232 & $\begin{array}{c}15 \\
(I C 95 \% 13,31- \\
16,86)\end{array}$ & $154(9,9)$ & $78(5,02)$ \\
\hline $\begin{array}{l}\text { Tuberculose pulmonar, baixo } \\
\text { risco }\end{array}$ & 229 & 14,75 & $151(9,72)$ & $78(5,02)$ \\
\hline $\begin{array}{l}\text { Tuberculose pulmonar, alto } \\
\text { risco }\end{array}$ & 1 & 0,06 & $1(0,06)$ & 0 \\
\hline $\begin{array}{l}\text { Tuberculose extra-pulmonar } \\
\text { (risco não apresentado) }\end{array}$ & 1 & 0,06 & $1(0,06)$ & 0 \\
\hline $\begin{array}{l}\text { Tuberculose mista (risco não } \\
\text { apresentado) }\end{array}$ & 1 & 0,06 & $1(0,06)$ & 0 \\
\hline
\end{tabular}

Fonte: elaborada pelos autores

\section{DISCUSSÃO}

Os resultados deste estudo mostraram elevada prevalência de indivíduos na faixa etária de 40 a 59 anos (33,23\%) cadastrados na UBSF. Em relação à estratificação por grupos de risco, a maior prevalência de indivíduos foi no grupo de Saúde integral da mulher (41,53\%), seguido pelos grupos de hipertensos $(29,36 \%)$, de crianças e adolescentes (22,41\%) e de tuberculose (15\%).

O presente estudo destaca o número considerável de crianças e adolescentes cadastrados nos serviços de saúde oferecidos pela unidade estudada. Apesar de serem classificadas em sua maioria como baixo risco, sabe-se que a infância, por ser uma fase de maior fragilidade do ser humano, é um período decisivo na formação do indivíduo. Os eventos ocorrentes nesse momento inicial da vida influenciam na formação do adulto futuro, considerando a introdução de estilos de vida que podem ser mantidos ao longo do tempo
(CARVALHO et al, 2015; ROCHA et al, 2016). Assim sendo, pensando nos benefícios de um diagnóstico precoce e monitoramento de saúde, o número de crianças que fazem uso da UBSF é considerado um aspecto positivo, uma vez que se torna possível um acompanhamento dos fatores de riscos aos quais essas crianças estão submetidas, evitando possíveis doenças ou agravos futuros.

Observa-se também o grande número de mulheres na amostra analisada ( $\mathrm{n}=1.097$; $70,6 \%)$, enfatizando os resultados já apresentados anteriormente pelo Ministério da Saúde (MS), que mostrou um número superior de indivíduos do sexo feminino nas pesquisas (BRASIL, 2016). No estudo de GUIBU et al (2017), que avaliou uma amostra de 8676 pessoas cadastradas na atenção primária à saúde, $75,8 \%$ eram mulheres, o que corrobora com o presente estudo, evidenciando que as mulheres buscam mais precocemente e com maior frequência as unidades de saúde do que os homens (GUIBU et al, 2017; MALTA, et al, 2017). 
Outro achado importante foi em relação ao número de indivíduos adultos, principalmente com idades mais avançadas $(33,23 \%)$ e idosos (18,80\%), direcionando a discussão para a mudança demográfica que a população brasileira vem passando nos últimos anos. A taxa de envelhecimento da população ultrapassa o número de nascimentos, implicando em características específicas do envelhecer dessa população com relação ao estado de saúde (CAVALCANTI, et al, 2009). O processo de envelhecimento traz consigo a resposta aos hábitos mantidos ao longo da vida, tais como a alimentação inadequada, sedentarismo, alto consumo de tabaco e álcool, levando ao aparecimento de diversas doenças crônicas condicionadas pelo estilo de vida (CAVALCANTI, et al, 2009), o que pode justificar a elevada prevalência de DCNT também encontradas no presente estudo.

Em relação às doenças mais prevalentes, quando somadas as porcentagens dos grupos com DM tipo I e tipo II, HA e DM tipo I e HA e DM tipo II, encontramos $10,75 \%$ de indivíduos com algum tipo de DM, o que se aproxima dos resultados apresentados pelo Sistema de Vigilância de Fatores de Risco e Proteção para Doenças Crônicas por Inquérito Telefônico (VIGITEL), que em 2018 relatou 7,7\% de indivíduos com DM (BRASIL, VIGITEL, 2018). Sobre a HA, no ano 2016, 37,34\% dos indivíduos tinham diagnóstico da doença, o que reduziu para 24,7\% em 2018 (BRASIL, VIGITEL, 2016; BRASIL, VIGITEL, 2018).

As DCNT são responsáveis por 41 milhões de mortes a cada ano, o equivalente a $71 \%$ de todas as mortes no mundo (OMS, 2018; BRASIL, 2011). No Brasil, segundo dados do sistema VIGITEL (BRASIL, 2016), entre os anos de 2006 e 2016, houve um aumento de $61,8 \%$ dos casos de DM e $14,2 \%$ dos casos de HA no país. Também se observou aumento de indivíduos com excesso de peso (passando de 42,6\% em 2006 para $55,7 \%$ em 2018), sendo que os casos de obesidade cresceram $60 \%$ em dez anos (BRASIL, VIGITEL, 2016).

O estilo de vida o qual o indivíduo segue pode elevar ou diminuir as chances da ocorrência de determinada doença. Quando se trata de DM e HA não é diferente, uma alimentação de má qualidade que resulte em deficiências ou excessos de alguns nutrientes, cursando com desequilíbrio metabólico e por vez na obesidade e dislipidemia, associadas ao sedentarismo e vícios (cigarro e bebidas alcoólicas) pode gerar como consequência o aumento dos níveis pressóricos e da glicose (MALTA, et al 2017). Além disso, os fatores de risco para DM e HA estão associados a aspectos socioeconômicos. Estudos recentes mostraram que o nível de escolaridade e a renda continuam sendo um grande desafio quando se trata de DCNT, uma vez que as consequências ligadas a essas condições estão diretamente relacionadas com os fatores de riscos os quais estes indivíduos estão expostos (CARVALHO, et al, 2016; LOBO, et al, 2017), o que justifica o fato da maioria dos indivíduos estarem classificados em médio e alto risco.

Quanto à tuberculose, encontramos uma prevalência de $15 \%$ na população estudada. A tuberculose é uma patologia de característica infectocontagiosa tendo como agente transmissor a bactéria Mycobacterium tuberculosis e Mycobacterium leprae. É uma doença que atinge o sistema imunológico, implicando os processos de defesa do organismo e tornando-o susceptível a infecções oportunistas, comprometimentos secundários e por consequência, aumenta a mortalidade do paciente (SILVA, 2018). Segundo o boletim Epidemiológico do Ministério da Saúde publicado em 2015, o Brasil faz parte do ranking mundial de países com alta prevalência de tuberculose (BRASIL, 2015). Um boletim mais recente comparou os números de casos de tuberculose no país nos anos de 2014 a 2018 e concluiu que, por mais que os índices da doença tenham apresentado uma diminuição por certo período, devido à implementação de políticas e programas que auxiliaram no diagnóstico e tratamento, nos últimos anos o número de casos voltou a aumentar podendo ser um dos resultados da atual economia do país, colocando a população em risco (BRASIL, 2019). Sabe-se que apresentam maiores riscos e predisposição dessa doença indivíduos que estão expostos a 
carências de fatores higiênicos e sanitários essenciais para vida diária, sendo que estas mesmas pessoas são as que, em geral, possuem menores condições socioeconômicas (OLIVEIRA, PETRONI, 2017). Outros fatores de risco relacionados à baixa renda e baixa escolaridade que elevam as chances de aquisição da tuberculose e/ou agravo do quadro se referem à alimentação não saudável, estilo de vida sedentário, o vício em tabaco, o que por sua vez compromete o sistema imunológico aumentando as chances de infecções (SILVA, et al, 2018).

Não menos importante, a hanseníase também classificada como doença crônica teve baixa prevalência neste estudo $(0,32 \%)$, reforçando os resultados da pesquisa de Ribeiro e colaboradores, que mostraram diminuição dos casos de hanseníase nos anos entre 2005 e 2015, prevendo queda ainda maior dos indicativos da doença nos anos subsequentes. Porém, a pesquisa enfatiza a importância de avaliação e monitoramento geográfico desses dados, pois os hábitos e cultura local são fortes indicativos à predisposição dessa patologia, além da influência das condições socioeconômicas regionais (RIBEIRO et al, 2018).

Por fim, a prevalência de transtornos mentais foi de $4,25 \%$. Os transtornos mentais vêm aumentando ao longo dos anos, pincipalmente aqueles cujos sintomas relacionados são de base depressiva e de ansiedade, muitas vezes podendo ter alguma ligação à situação socioeconômica do indivíduo ou da família (MARAGNO et al., 2006). Visto que a saúde psíquica em risco pode levar a alterações fisiológicas e físicas, a ansiedade pode ser um dos fatores de risco por propiciar baixa qualidade de vida que resulta no surgimento de algumas doenças, inclusive as crônicas (COSTA et al., 2019).

Tendo em vista os achados deste estudo, fica evidente a importância do papel governamental na elaboração e fortalecimento de políticas públicas, que visem à promoção e o monitoramento em saúde. Em síntese, se faz necessária a elaboração de estratégias que abordem o contexto socioeconômico, demográfico e biológico dos indivíduos, de forma a promover um atendimento acolhedor e humanizado, valorizando a escuta aos anseios e dificuldades enfrentadas pelo paciente no dia a dia, disseminando informações a respeito de qualidade de vida no seu amplo significado, garantindo o direito a um acompanhamento de maior qualidade.

\section{CONSIDERAÇÕES FINAIS}

Os achados do presente estudo evidenciaram uma elevada prevalência de indivíduos cadastrados na UBSF com faixa etária de 40 a 59 anos, do sexo feminino e com doenças crônicas, especialmente HA, DM (alto risco familiar) e tuberculose. Destaca-se a importância do papel governamental na elaboração e fortalecimento de políticas públicas, que visem à promoção e monitoramento das condições de saúde na APS. 


\section{REFERÊNCIAS}

BRASIL. Ministério da Saúde. Brasil Livre da Tuberculose: evolução dos cenários epidemiológicos e operacionais da doença. Secretária de Vigilância em Saúde: Boletim Epidemiológico, 2019. Brasília, v.50, n.9 Disponível em: https://portalarquivos2.saude.gov.br/images/pdf/2019/marco/22/2019-009.pdf. Acesso em: 18 de set. 2019.

BRASIL. Ministério da Saúde. Detectar, tratar e curar: desafios e estratégias brasileiras frente à tuberculose. Secretaria de Vigilância em Saúde: Boletim Epidemiológico, 2015. Brasília, v.46, n.9 Disponível em: http://portalarquivos.saude.gov.br/images/pdf/2015/marco/25/Boletim-tuberculose-2015.pdf

BRASIL. Ministério da Saúde. Secretaria de Atenção à Saúde. Departamento de Atenção Básica. Estratégias para o cuidado da pessoa com doença crônica / Ministério da Saúde, Secretaria de Atenção à Saúde, Departamento de Atenção Básica. - Brasília: Ministério da Saúde, 2014.

BRASIL. Ministério da Saúde. Secretaria de Atenção à Saúde. Departamento de Atenção Básica. Política Nacional de Atenção Básica / Ministério da Saúde. Secretaria de Atenção à Saúde. Departamento de Atenção Básica. - Brasília: Ministério da Saúde, 2012.

BRASIL. Ministério da Saúde. Vigitel: Hábitos dos brasileiros impactam no crescimento da obesidade e aumenta prevalência de diabetes e hipertensão, 2016. Disponível em: <http://portalarquivos.saude.gov.br/images/pdf/2017/abril/17/Vigitel.pdf. Acesso em 14 de dez. 2018.

BRASIL. Ministério da Saúde: Portal do Governo Brasileiro, Plano de Ações Estratégias para o Enfrentamento das Doenças não Transmissíveis (DCNT), 2011. Disponível em: http://www.saude.gov.br/vigilancia-em-saude/vigilancia-de-doencas-cronicas-nao-transmissiveis$\mathrm{dcnt} /$ plano-de-acoes-estrategicas-para-o-enfrentamento-das-doencas-cronicas-nao-transmissiveis-dcnt. Acesso em 24 de nov. 2019.

BRASIL. Ministério da Saúde: Portal do Governo Brasileiro. Política Nacional de Vigilância em Saúde, 2018. Disponível em: http://www.saude.gov.br/vigilancia-em-saude/politica-nacional-de-vigilancia-em-saude. Acesso em 19 de set. 2019.

CARVALHO, C. A. et al. Consumo alimentar e adequação nutricional em crianças brasileiras: revisão sistemática. Revista Paulista de Pediatria, São Paulo, v. 32, n.2, p.211-221, jun. 2015. Disponível em: https://www.sciencedirect.com/science/article/pii/S0103058215000234. Acesso em: 18 de set. 2019.

CARVALHO, C. J. et al. Altas taxas de sedentarismo e fatores de risco cardiovascular em pacientes com hipertensão arterial resistente. Medicina (ribeirão Preto. Online), Ribeirão Preto, v. 49, n. 2, p.124-133, 2 abr. 2016. Universidade de São Paulo Sistema Integrado de Bibliotecas - SIBiUSP. 2176-7262. v. 49i2. P. 124-133.

CAVALCANTI. C, L. et al. Prevalência de doenças crônicas e estado nutricional em um grupo de idosos brasileiros. Revista Salud Pública. Paraíba, v.11, n. 6, p.865-877, nov. 2009. Disponível em: https://www.scielosp.org/pdf/rsap/2009.v11n6/865-877/pt. Acesso em 18 de set. 2019.

COSTA, C. O. et al. Prevalência de ansiedade e fatores associados em adultos. Jornal Brasileiro de Psiquiatria. São Paulo, v.68, n.2, p. 92-100, jul. 2019. Disponível em: http://www.scielo.br/pdf/jbpsiq/v68n2/1982-0208-jbpsiq-68-02-0092.pdf. Acesso em: 18 de set. 2019.

DATASUS. Tecnologia da Informação a Serviço do SUS. Disponível em: http://tabnet.datasus.gov.br/cgi/tabcgi.exe?siab/cnv/SIABSMG.def. Acesso em: 23/01/2018. 
GUIBU, I. A. et al. Características principais dos usuários dos serviços de atenção primária à saúde no Brasil. Revista de Saúde Pública. São Paulo, v.51. n.2, set. 2017. Disponível em: http://www.rsp.fsp.usp.br/artigo/caracteristicas-principais-dos-usuarios-dos-servicos-de-atencaoprimaria-a-saude-no-brasil/ Acesso em: 18 de set. 2019.

IBGE. Instituto Brasileiro de Geografia e Estatística. Disponível em: https://cidades.ibge.gov.br/brasil/mg/uberlandia/panorama. Acesso em: 23/01/2018.

LOBO, L. A. C. et al. Tendência temporal da prevalência de hipertensão arterial sistêmica no Brasil. Cadernos de Saúde Pública, Porto Alegre, v. 33, n. 6, p.33-38, 28 jul. 2017. Disponível em: https://www.scielosp.org/pdf/csp/2017.v33n6/e00035316/pt. Acesso em: 15 de ago. 2019.

MALTA, D. C. et al. Prevalência e fatores associados à hipertensão autorreferida em adultos brasileiros. Rev. Saúde Pública, São Paulo, v. 51, 2017. Disponível em: http://www.scielo.br/scielo.php?script=sci_arttext\&pid=S003489102017000200313\&lng=en\&nrm=iso. Acesso em: 15 de agos. 2019.

MARAGNO, L. et al. Prevalência de transtornos mentais comuns em populações atendidas pelo Programa Saúde da Família (QUALIS) no Município de São Paulo, Brasil. Caderno de Saúde Pública. Rio de Janeiro, v.22, n.8. p. 1639-1648, ago. 2006. Disponível em: https://www.scielosp.org/pdf/csp/2006.v22n8/16391648/pt Acesso em 18 de set. 2019.

MENDES, E.V. O cuidado das condições crônicas na atenção primária à saúde: o imperativo da consolidação da estratégia da saúde da família. / Eugênio Vilaça Mendes. Brasília: Organização Pan-Americana da Saúde, 2012. 512 p.: il.

OLIVEIRA, G. O; PETRONI, T. F. Avaliação de Indicadores Epidemiológicos da Tuberculose no Brasil. Revista Saúde Unitoledo, Araçatuba, v. 01, n. 01, p.134-146, 01 ago. 2017. Disponível em: http://ojs.toledo.br/index.php/saude/article/view/2443/108. Acesso em: 15 de ago. 2019.

ORGANIZAÇÃO MUNDIAL DA SAÚDE (OMS). Time to deliver: report of the WHO Independent High-Level Commission on Noncommunicable Diseases. 2018. Disponível em: https://apps.who.int/iris/bitstream/handle/10665/272710/9789241514163-eng.pdf?ua=1. Acesso em: 02 de set. 2019.

PLANO DIRETOR DA ATENÇÃO PRIMÁRIA À SAÚDE. Secretaria de Estado de Saúde de Minas Gerais e Escola de Saúde Pública de Minas Gerais, 2010.

RIBEIRO, M. D. et al. Estudo Epidemiológico da hanseníase no Brasil: reflexão sobre as metas de eliminação. Revista Panam Salud Pública. São Paulo, v.1, n.1, p.42-49, fev. 2018. Disponível em: http://iris.paho.org/xmlui/bitstream/handle/123456789/34882/v42e422018.pdf?sequence=1\&isAllowed =y. Acesso em 18 de set. 2019.

ROCHA, N. P. et al. Associação de insegurança alimenta e nutricional com fatores de risco cardiometabólicos na infância e adolescência: uma revisão sistemática. Revista Paulista de Pediatria. São Paulo, v.34, n.2, p.225-233, jun.2016. Disponível em: https://www.sciencedirect.com/science/article/pii/S0103058215001252. Acesso em: 18 de set. 2019.

SECRETARIA de Saúde. Prefeitura de Uberlândia: Programa de Educação Permanente - PEP, UBSF Jardim Brasília 2, 2018.

SILVA, D. R. et al. Fatores de risco para tuberculose: diabetes, tabagismo, uso de álcool e uso de outras drogas. J. bras. pneumol., São Paulo, v. 44, n. 2, p. 145-152, Abril, 2018b. Disponível em: 
http://www.scielo.br/scielo.php?script=sci_arttext\&pid=S1806-37132018000200145\&lng=en\&nrm=iso Acesso em: 15 de agos. 2019.

SILVA, M. E. N. et al. Aspectos gerais da tuberculose: uma atualização sobre o agente etiológico e o tratamento. Rbac, Fortaleza, v. 3, n. 50, p.228-232, 06 nov. 2018a. Disponível em: <http://www.rbac.org.br/wp-content/uploads/2019/01/RBAC-vol-50-3-2018-ref-717-final.pdf>. Acesso em: 15 de agos. 2019. 